\title{
Cardiovascular Changes in Children with Acute Lower Respiratory Tract Infection
}

\author{
SHAHREEN KABIR ${ }^{1}$, SOOFIA KHATOON ${ }^{2}$, LUTFUL EHSAN FATMI ${ }^{3}$, NAZNEEN AKHTER BANU ${ }^{4}$, ARIF \\ AHMED MOHIUDDIN ${ }^{5}$, RUBINAAFROZ RANA ${ }^{6}$
}

\begin{abstract}
Background: Acute Lower Respiratory Tract Infections (ALRI), particularly Pneumonia and Bronchiolitis, are important causes of death in childhood in Bangladesh. The cardiovascular and respiratory systems function as a single unit and alteration in cardiorespiratory interactions, can cause significant changes in cardiac function. The objective of this study was to find out any electrical and functional changes, myocardial injury, frequency of heart failure and the outcomes in these patients with ALRIs.
\end{abstract}

Methodology: It was a prospective observational study carried out at DMCH from January to June 2012 on 35 consecutive children admitted with ALRI, which were further diagnosed as pneumonia or bronchiolitis using operational definitions. Heart failure cases were identified and all the cases were then evaluated for any cardiovascular changes.

Results: Most of the patients were male. ECG changes occurred in the form of tachycardia. Abnormal echocardiographic findings were noted in the form of pulmonary hypertension, left ventricular systolic (LV) dysfunction and tricuspid regurgitation. $82.9 \%$ had raised $C K-M B$ and was significantly higher in patients with tachycardia and having abnormal echocardiographic changes. Nine patients developed heart failure, 3 of them had pulmonary hypertension along with LV systolic dysfunction and one of pulmonary hypertension and LV systolic dysfunction each. $C K-M B$ was raised significantly in all the patients with heart failure.

Conclusion: Raised CK-MB, tachycardia out of proportion on ECG and pulmonary hypertension with left ventricular systolic dysfunction were common findings in patients with ALRI.

Keywords: Cardiovascular, CK-MB, Pneumonia, Bronchiolitis.

\section{Introduction:}

Acute lower respiratory tract infection (ALRI) particularly pneumonia is an important cause of death

1. Registrar, Pediatric Cardiology, National Heart Foundation and Research Institute, Darus-salam, Dhaka.

2. Academic coordinator and Head, Department of Pediatrics, $\mathrm{CWCH}$, Ashulia, Dhaka.

3. Head of Department of Pediatrics, Holy Family Medical College and Hospital, Dhaka.

4. Head of Department of Pediatrics, Sir Salimullah Medical College and Mitford Hospital, Dhaka.

5. Specialist, Department of Cardiac Surgery, United Hospital Limited, Gulshan, Dhaka

6. Jr. Consultant (Pediatrics), NCCRFHD, Sher-E-Bangla Nagar, Dhaka.

Correspondence: Dr. Shahreen Kabir, Registrar, Pediatric Cardiology, National Heart Foundation and Research Institute, Darus-salam, Dhaka. E-mail: ninia_k@hotmail.com Mobile: +8801971566834

Received: 19-12-2018

Accepted: 16-03-2019 in childhood. ${ }^{1}$ Acute respiratory tract infection (ARI) can be classified into upper and lower respiratory tract infection. Structures inferior to the vocal cord are accounted as lower respiratory tract. Among many, Pneumonia and Bronchiolitis remain the most important cause of respiratory illness in Bangladesh. In Bangladesh about $21 \%$ and $11.5 \%$ of under five children attending hospital are diagnosed as bronchiolitis and bronchopneumonia respectively. ${ }^{2}$ The cardiovascular and respiratory systems function as a single unit and alteration in cardiorespiratory interactions, can cause significant changes in cardiac function..$^{1,3}$ Pneumonia can cause myocarditis by direct invasion of the microorganism, can lead to cardiac failure by myocardial depressant effect of hypoxia, and may further deteriorate cardiac function by altering preload and after load. ${ }^{1,3,4}$ The main cause 
of acute pulmonary hypertension is hypoxia that leads to pulmonary vasoconstriction, causing right ventricular dysfunction. ${ }^{5}$ Pulmonary hypertension increases the wall stress of right ventricle, results is a greater demand for coronary blood flow which is generally reduced because of inadequate diastole. Pulmonary hypertension causes right ventricular pressure over load and hypertrophy. The enlarged right ventricle eventually shifts the interventricular septum towards the left and limits left ventricular filling and output causing left ventricular dysfunction. $6,7,8$ Since myocarditis can be a fatal disease, its detection is important by non-invasive techniques like ECG, Echocardiography and cardiac enzyme measurements. ${ }^{1,9}$ although the gold standard of its detection remains by cardiac muscle biopsy nevertheless avoided due its invasive nature ${ }^{1}$. The different cardiac enzymes available for detecting myocarditis are CK-MB, Troponin I and $\alpha-\mathrm{HBDH} .{ }^{1,10,11}$ $\mathrm{CK}-\mathrm{MB}$ is considered as a strong marker for myocardial damage and its rise may be the only sign of subclinical myocarditis. ${ }^{12}$ Although Troponin I is much more accurate and specific in detecting myocarditis in acute coronary syndrome, its rise is also detected in non-cardiac causes. ${ }^{13,14} \mathrm{~A}$ rise of pro-BNP is also highly significant for pediatric heart failure ${ }^{15}$, which along with áHBDH measurement was not available in this hospital setting. So the remaining choice was measurement of CK-MB which is a specific cardiac enzymes for the detection of myocardial injury ${ }^{1}$.Cardiac failure is a serious complication of pneumonia in developing countries, like Bangladesh, and may also contribute to mortality, but its role has not been clearly defined by studies in this country. ${ }^{1,9,16}$ The objective of the study was to find out if there was any electrical, functional changes, myocardial injury, the frequency of heart failure and the outcomes in these patients with ALRIs.

\section{Materials and Method}

An observational case series comprised of 35 consecutive children admitted with acute lower respiratory infection at Department of Paediatrics was carried out at Dhaka Medical College and Hospital from January to June in the year 2012. Children aged less than 2 months and more than 5 years, or having severe acute malnutrition, or having congenital heart disease or previously diagnosed with bronchial asthma were excluded from the study. Initially 50 consecutive patients fulfilling the inclusion criteria were taken into the study. Out of these, six were taken out of the study as they deteriorated and became too sick to be evaluated, ultimately 35 were fully assessed. Written consents were taken from all the parents. Detailed history was taken and noted in the pre-tested questionnaire. Thorough examination of the respiratory, cardiovascular and other related systems was carried out and diagnosed as either pneumonia or bronchiolitis according to the case definition. Blood samples were taken on admission for complete blood count, serum electrolytes, C reactive protein and CK-MB. Cardiovascular workup such as X-ray chest, ECG and Echocardiography were carried out. Patients were managed according to the institutional protocol with oxygen inhalation as per requirement, proper antibiotics in cases of pneumonia, Digoxin to those who developed heart failure. The cardiovascular workups were compared in the pneumonia and bronchiolitis group. Patients who developed heart failure were identified and their results were compared with patients who did not. Statistical analyses related were performed by using of SPSS 17.0 package program.

\section{Results}

Out of the 35 patients, $82.5 \%$ were between the age of 2 months to 6 months and $80.1 \%$ were male. Depending on clinical features, auscultatory findings and chest x-ray findings, 15 (42.9\%) children was diagnosed as Bronchopneumonia and 20 (57.1\%) as Bronchiolitis. They were further evaluated by ECG, Echocardiography and CK-MB for cardiovascular changes.

The ECGs showed tachycardia in $10(66.7 \%)$ patients with Bronchopneumonia and $9(45 \%)$ with Bronchiolitis although the difference was not statistically significant. None of them had arrhythmia (Table-I).

Table I

Distribution of patients by ECG findings ( $n=35$ )

\begin{tabular}{lccc}
\hline ECG findings & $\begin{array}{c}\text { Pneumonia } \\
(\mathrm{n} \%)\end{array}$ & $\begin{array}{c}\text { Bronchiolitis } \\
(\mathrm{n} \%)\end{array}$ & $\begin{array}{c}\mathrm{P} \\
\text { value }\end{array}$ \\
\hline Normal & $5(33.3)$ & $11(55.0)$ & \\
Tachycardia & $10(66.7)$ & $9(45.0)$ & $0.202^{\text {ns }}$ \\
Arrhythmia & 0.0 & 0.0 & \\
\hline
\end{tabular}

ns= not significant

$P$ value reached from chi-square test 
At echocardiographic evaluation (Table-II), it was seen $15(75 \%)$ from the Bronchiolitis group had normal findings whereas $4(26.7 \%)$ patients from pneumonia group had normal findings, the rest of the patients from the Pneumonia group, 11 (73.3\%), had abnormal findings, i.e there were more echocardiographic changes in the pneumonia group. However, Pulmonary hypertension was noted in 5 $(33.3 \%)$ and $3(15 \%)$, LV systolic dysfunction in 6 $(40 \%)$ and $6(30 \%)$ in Bronchopneumonia and Bronchiolitis group respectively, Tricuspid Regurgitation was found in $2(13.3 \%)$ children with Bronchopneumonia and 1(5\%) with Bronchiolitis.

Table II

Distribution of the study patients by echocardiographic findings $(n=35)$

\begin{tabular}{lccc}
\hline $\begin{array}{l}\text { Echocardiographic } \\
\text { findings }\end{array}$ & $\begin{array}{c}\text { Pneumonia } \\
(\mathrm{n}=15)\end{array}$ & $\begin{array}{c}\text { Bronchiolitis } \\
{ }^{*}(\mathrm{n}=20)\end{array}$ & $\begin{array}{c}\mathrm{P} \\
\text { value }\end{array}$ \\
\hline Normal & $\mathrm{n}(\%)$ & $\mathrm{n}(\%)$ & \\
Pulmonary hypertension & $5(26.7)$ & $15(75.0)$ & ${ }^{\mathrm{a}} 0.004^{\mathrm{s}}$ \\
LV systolic dysfunction & $6(40.0)$ & $3(15.0)$ & ${ }^{\mathrm{a}} 0.191^{\mathrm{ns}}$ \\
Tricuspid regurgitation & $2(13.3)$ & $1(5.0)$ & ${ }^{\mathrm{a}} 0.390^{\mathrm{ns}}$ \\
\hline
\end{tabular}

* A single patient may exhibit multiple findings

CK-MB values are marked normal upto 25U/L. 82.9\% patients were found to have raised CK-MB levels (Figure-1). Mean CK-MB difference was not statistically significant $(P>0.05)$ between bronchopneumonia and bronchiolitis, i.e CK-MB was raised irrespective of the diagnosis (Table-III).

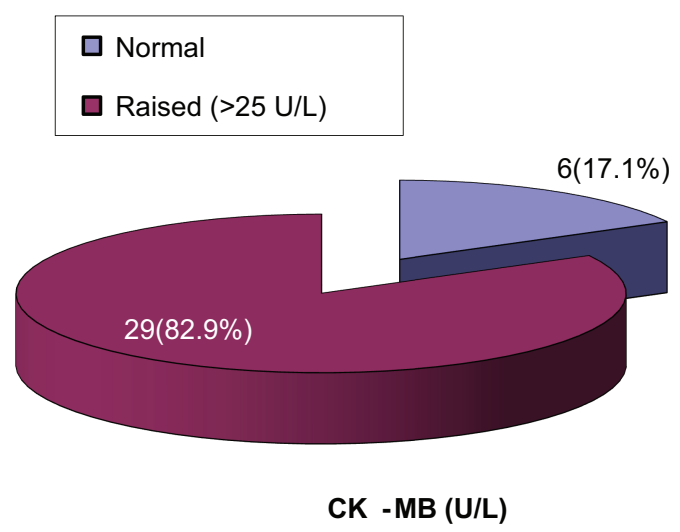

Fig 1: Distribution of patients by CK-MB findings ( $n=35)$

\section{Table III}

Distribution of study patients with mean CKMB value by diagnosis $(n=35)$

\begin{tabular}{lcccc}
\hline & \multicolumn{3}{c}{ CKMB (U/L) } & P value \\
\hline Diagnosis & No. & Mean $\pm S D$ & (Min-max) \\
Bronchopneumonia & 15 & $41.46 \pm 17.42$ & $(17-66)$ & $0.801^{\text {ns }}$ \\
Bronchiolitis & 20 & $40.1 \pm 14.26$ & $(10-71)$ \\
\hline
\end{tabular}

Figure-2 showed 9 (25\%) patients, among all 35 developed heart failure, 6 of which had Bronchiolitis and 3 pneumonia. Table-IV showed the cardiovascular workup of the heart failure group. All of them had tachycardia on ECG. In echo-cardiography among the 9 patients, 4(44.4\%) had normal findings and the rest $5(56 \%)$ had findings. $3(33.3 \%)$ of them had Pulmonary hypertension along with LV Systolic Dysfunction, 1 of pulmonary hypertension and LV systolic dysfunction each. All of them had raised CKMB levels.

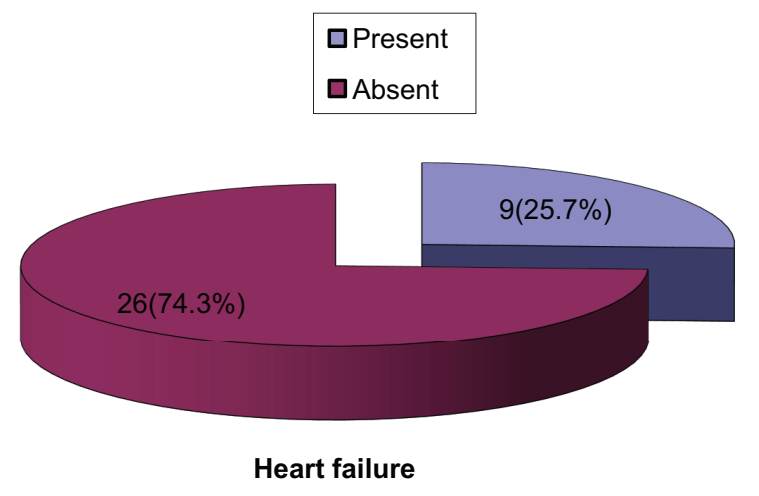

Fig 2: Distribution of the study patients by presence and absence of heart failure $(n=35)$

(81\%). Pulmonary hypertension and LV systolic dysfunction findings by Echocardiography was also found to be significantly different $(P<0.05)$ between the normal and tachycardic groups signifying that patients with tachycardia on ECG had more Pulmonary hypertension and LV systolic dysfunction than those with normal ECG.

Patients with normal Echo findings had more normal ECG findings (Table-V). All the patients with Pulmonary hypertension had tachycardia on ECG and LV systolic dysfunction was significantly more in patients with tachycardia $(\mathrm{p}<0.5)$.

Mean CK-MB was found to be higher in tachycardic group than those with normal heart rate and the 
difference was statistically significant $(P<0.05)$. Mean CK-MB was also raised significantly more in patients with pulmonary hypertension, LV systolic dysfunction and in patients with Tricuspid regurgitation than patients with normal echo findings (Table-VI). They were also statistically significant. Table-VII showed association of heart failure with $\mathrm{CK}-\mathrm{MB}$ value. Mean CKMB was found to be $50.33 \pm 11.6 \mathrm{U} / \mathrm{L}$ in with heart failure and $36.72 \pm 14.9 \mathrm{U} / \mathrm{L}$ in without heart failure. The difference was statistically significant $(P<0.05)$ between two groups. This means $\mathrm{CK}-\mathrm{MB}$ value was significantly raised in heart failure group. All the patients survived, the patients who developed heart failure stayed around 9 to days and those did not were discharged with an average of 5 days.
Table IV

Distribution of heart failure patients by cardiovascular workup $(n=9)$

\begin{tabular}{lc}
\hline Cardiovascular Workup & $\mathrm{n}(\%)$ \\
\hline ECG: Tachycardia & $9(100 \%)$ \\
ECHO: & \\
Pulmonary Hypertension with LV & $3(33.3 \%)$ \\
Systolic Dysfunction & \\
Pulmonary Hypertension & $1(11.1 \%)$ \\
LV Systolic Dysfunction & $1(11.1 \%)$ \\
Normal & $4(44.4 \%)$ \\
Mean CK-MB & 50.33 \\
\hline
\end{tabular}

Table V

Distribution of the patients with ECG findings by Echocardiographic changes $(n=35)$

\begin{tabular}{lccccc}
\hline \multirow{2}{*}{ Echocardiographic changes } & \multicolumn{4}{c}{ ECG Findings } & P value \\
& Normal $(\mathrm{n}=16)$ & \multicolumn{2}{c}{ Tachycardia $(\mathrm{n}=19)$} & \\
\hline Normal & $\mathrm{N}$ & $\%$ & $\mathrm{~N}$ & $\%$ & \\
Pulmonary hypertension & 13 & 81.2 & 6 & 31.6 & $0.003^{\mathrm{s}}$ \\
LV systolic dysfunction & 0 & 0.0 & 8 & 42.1 & $0.003^{\mathrm{s}}$ \\
Tricuspid regurgitation & 2 & 12.5 & 10 & 52.5 & 0.012 \\
\hline
\end{tabular}

* A single patient may exhibit multiple findings

Table VI

Distribution of study patients with mean CK-MB value by ECG and Echocardiographic findings ( $n=35)$

\begin{tabular}{llcccc}
\hline & No. & CKMB (U/L) & & P value \\
& Normal & Mean \pm SD & (Min-max) & \\
\hline ECG findings & Tachycardia & 16 & $34.5 \pm 15.24$ & $(10-71)$ & $0.043^{s}$ \\
& Normal & 19 & $44.61 \pm 13.22$ & $(20-66)$ & \\
& Pulmonary hypertension & 8 & $34 \pm 12.4$ & $(10-60)$ & $0.036^{\text {s }}$ \\
& LV Systolic dysfunction & 12 & $45 \pm 16.28$ & $(20-62)$ & \\
& Tricuspid regurgitation & 3 & $49.33 \pm 12.22$ & $(29-62)$ & \\
& & & & & \\
\hline
\end{tabular}

Table VII

Distribution of study patients with and without heart failure by CK-MB findings ( $n=35)$

\begin{tabular}{lccccc}
\hline & \multicolumn{3}{c}{ With heart failure $(\mathrm{n}=9)$} & \multicolumn{3}{c}{ Without heart failure $(\mathrm{n}=25)$} & P value \\
& Mean & $\pm \mathrm{SD}$ & Mean & $\pm S \mathrm{D}$ & \\
\hline CKMB (U/L) & 50.33 & \pm 11.6 & 36.72 & \pm 14.9 & $0.018^{\mathrm{s}}$ \\
Range (min-max) & $(30$ & $-66)$ & $(10$ & $-71)$ & \\
\hline
\end{tabular}




\section{Discussion}

ALRI is an important cause of death in childhood in Bangladesh. The functioning of the cardiovascular and the respiratory systems are dependent on each other in various ways and alteration in their interactions, can cause significant changes in cardiac function ${ }^{1}$. This study was carried out on 35 children with ALRI aging from 2 to 59 months and was diagnosed as Bronchiolitis in 20 (57.1\%) patients and Bronchopneumonia in 15 (42.9\%) patients.

Tachycardia was observed in $66.7 \%$ and $45 \%$ in the Pneumonia and Bronchiolitis group respectively by ECG in the current study and the results were not significantly different from each other. No arrhythmia was observed in the study patients (Table-I). Similar findings were seen by Fine et al ${ }^{17}$ in $26 \%$ of the study population, and $64.5 \%$ by Kocak et al. ${ }^{18}$ Fine et al stated that the most significant single sign of myocarditis was a tachycardia out of proportion to temperature. Another study by Esposito et al found an association with sinoatrial block and RSV load implementing RSV could play a direct role in inducing arrhythmia. ${ }^{10}$

The Echocardiographic findings in this study revealed Pulmonary hypertension in 33.3\% in Pneumonia and $15 \%$ in Bronchiolitis group, Left ventricular systolic dysfunction in $40 \%$ and $30 \%$ in Pneumonia and Bronchiolitis group respectively (Table-II). It is to be noted that abnormal findings were more common in the pneumonia group, although difference in findings between the two groups were not stastically significant. Bardi-Peti ${ }^{19}$ et al found Pulmonary hypertension in 18 patients with acute respiratory disease; they however interestingly described the mechanical effect of hyperinflation of the lung parenchyma on pulmonary vessels to be the probable cause of increased pulmonary arterial pressure during these broncho-obstructive diseases. Tricuspid regurgitation is a common finding along with pulmonary hypertension, although it can be found isolated in normal patients. Isolated TR was found in a study by Senocak et $\mathrm{al}^{1,20}$ as a part of normal cardiac anatomy and Neubauer et $\mathrm{al}^{21}$, Mahmoud et $\mathrm{al}^{22}$ along with reduction of ejection fraction and Sreeram et al ${ }^{19}$ found 11 patients with Bronchiolitis having TR in an otherwise normal cardiac finding. This current study, however, did not find right ventricular systolic dysfunction as a result of pulmonary hypertension, but rather left ventricular/r dysfunction which correlates with the study of Kumar ${ }^{6}$, Hopkins ${ }^{7}$ and Bates $^{8}$, where they signify that the enlarged right ventricle shifts the interventricular septum towards the left and causes left ventricular systolic dysfunction.

Serum CK-MB was found to be elevated in $82.9 \%$ subjects in this study in the absence of severe hypoxia, acidosis or sepsis (Figure-1). The finding was consonant with the $68 \%$ in the study by llten et $\mathrm{al}^{1}$ and with $60 \%$ of Mahmoud et $\mathrm{al}^{21}$ and significant increase by Ma et al ${ }^{38}$. Also, to be noted is the finding that CK-MB was raised unequivocally in both the bronchopneumonia and bronchiolitis groups (Table-III).

$9(25 \%)$ children developed heart failure (Figure-2). This was akin to the incidence $(14 \%)$ in the study by Ilten et $\mathrm{al}^{1}$ and $8.5 \%$ to that of Navarro et al ${ }^{23}$. All the patients had tachycardia on ECG (Table-IV). On Echocardiogram four patients had Pulmonary Hypertension out of which 3 were along with LV systolic dysfunction. One patient had only LV systolic dysfunction. All the patients had raised CK-MB level. Seedat et $a^{16}$ found tachycardia to be a poor prognostic feature among the patients with pneumonia who had ECG changes, especially those with a raised CK-MB level whereas Douglas et al ${ }^{24}$ concluded that uncontrolled tachycardia may result in significant LV dysfunction. Nerheim et al ${ }^{25}$ found that tachycardia-induced cardiomyopathy develops slowly and appears reversible by left ventricular ejection fraction improvement, but recurrent tachycardia causes rapid decline in left ventricular function and development of heart failure. Both these studies points out to the fact that tachycardia is associated with left ventricular dysfunction which is a prominent finding among the heart failure patients of the current study.

The most prominent echocardiographic finding of the heart failure patients in the prevailing study were Pulmonary hypertension along with LV systolic dysfunction (Table-IV). Although right heart involvement is a more common finding in patients with pulmonary hypertension, especially with respiratory tract infections as found by Shann et al ${ }^{5}$ where $26 \%$ of the subjects developed right ventricular failure secondary to pulmonary hypertension, but this current study interestingly found left ventricular involvement. These findings can be related to the study carried out by Shah et $\mathrm{al}^{26}$, who stated that Pulmonary hypertension is found secondary to left 
ventricular systolic dysfunction in $68 \%$ to $78 \%$ of patients with heart failure patients along with right heart involvement ${ }^{27,28,29}$. A different study was carried out to find out the determinants of pulmonary hypertension in left ventricular dysfunction by Enriques-Sarano et $\mathrm{al}^{30}$ and they concluded that pulmonary hypertension is associated with congestive heart failure. They discussed the probable causes to be an increase in left atrial pressure ${ }^{31}$ and pulmonary resistance ${ }^{32}$ and, possibly, from the loss of endothelium-dependent vasodilation of the pulmonary arterial bed ${ }^{33}$. Pulmonary hypertension is also associated with neurohumoral activation ${ }^{34,35}$ in particular of endothelin- $1^{36}$, a potent vasoconstrictor that is increased markedly in heart failure ${ }^{37}$. The probable cause of the left ventricular involvement can be attributed to myocarditis. ${ }^{38}$

All the children who developed heart failure probably had myocarditis as CK-MB is considered as a strong marker for myocardial damage and its rise may be the only sign of subclinical myocarditis as found by Heikkila et $\mathrm{al}^{37}$. In this current study CK-MB was also found to be significantly raised in children who had tachycardia on ECG ( $p=0.43)$ ( Table-VI). Similar finding of $26.1 \%$ of study group with tachycardia was associated with clinical myocarditis as found by Fine et $\mathrm{al}^{17}$. When seen the association between the CK$M B$ values with the Echocardiographic findings in the prevailing study, it was found to be more raised in those with Pulmonary hypertension, LV systolic dysfunction and Tricuspid regurgitation $(p=0.03)$ (Table-VI). This means that CK-MB values were much higher in children with abnormal echocardiographic findings than with children with normal findings ( $P$ value 0.036 ). Similar findings can be related to the study by Mahmoud et al. ${ }^{22}$

The most remarkable finding in this study found when the CK-MB values were compared between the heart failure and non-heart failure group. (TableVII). Serum CK-MB values were significantly high in those with heart failure than those without $(p=0.018)$, suggesting that children who developed heart failure also had myocarditis as depicted by the very high values of serum CK-MB in them.

\section{Conclusion}

Cardiovascular changes, along with heart failure were found in children with acute lower respiratory tract infections. Raised CK-MB, tachycardia out of proportion on ECG and pulmonary hypertension with left ventricular systolic dysfunction were common findings. Serum CK-MB, was raised in those with ECG and Echo changes. raised significantly more in the heart failure group. Cardiovascular changes in ALRI are associated with increased hospital stay and thus bed occupancy.

\section{References}

1. Ilten $F$, Senocak $F$,Zorlu P, Tesic T. Cardiovascular Changes in children with pneumonia, The Turkish journal of Pediatrics 2003; 45: 306-310

2. Kabir ARM. Report on magnitude of Respiratory disorder in under five children attending in different hospital in Bangladesh, BMRC report 2009, Dhaka.

3. Meliones JN, Cheifetz IM. Pulmonary physiology ad heart-lung interactions. In: Garson A, Bricker JT, Fishe DJ, Neish SR( eds). The Science and Practise of Pediatric Cardiology ( $2^{\text {nd }}$ edition). Vol. 1. Baltimore : Williams\& Wilkins; 1998: 279312

4. Navarro EE, Gonzaga NC, Lucero MG, Queipo SC, Gomez LO, Tupasi TE. Clinicopathologic studies of children who die of acute lower respiratory tract infections: mechanisms of death. Rev Infect Dis 1990; 12: 1065-1073

5. Shann F, Macgregor D, Richens J, Coakley J. Cardiac failure in children with pneumonia in Papua New Guinea. Pediatr Infect Dis J. 1998 Dec; 17(12): 1141-1143

6. Haworth S.G, Kumar R. K. Pulmonary Hypertension, India, Elsevier, 117-119

7. Hopkins WE. The remarkable right ventricle in patients with Eisenmenger syndrome. Coron Artery Dis 2005;16:19-25

8. Bates B, Bickley LS, Hoekelman RA. A Guide to Physical Examination and history Taking $6^{\text {th }}$ ed. Philadelphia: Lippincott; 1995

9. Kleigman RM, Stanton B, St.GemellI JW, Schor $\mathrm{N}$, Behrman RE. Nelson Textbook of Pediatrics, $19^{\text {th }}$ edition, Saunders, Philadelphia pg-1445

10. S. Esposito, P. Salice, S. Bosis. Altered cardiac rhythm in infants with bronchiolitis and respiratory syncytial virus infection. BMC infectious disease. 2010; 10:305 
11. Ma P, Guo L, Xia L. Clinical significance of the changes of heart function and myocardial enzyme in infantile pneumonia patients complicated with heart failure. Shangdong Medical Journal 2003-19

12. Heikkila J, Karjaleinen J. Evaluation of mild acute infectious myocarditis. Br Heart J 1982; 47: 381-391.

13. Higgins J, Seward J. Elevation of cardiac Troponin I indicates more than myocardial ischaemia. Clin Invest Med 2003; 26(3): 133-147

14. Korff S, Katus Hugo, Giannitsis E. Differential diagnosis of elevated troponins. Heart 2006; 92(7): 987-993

15. Chun-Wang L, Xiang-Lin Z, Shao-Hu J. Role of the NT-proBNPlevel in the diagnosis of pediatric heart failure and investigation of novel combined diagnostic criteria. Experimental and therapeutic medicine 2013; 6(4):995-999

16. Seedat MA, Feldman C, Skoularigis J, Prommnitz DA, smith C, Zwi S. A study of acute community-acquired pneumonia, including details of cardiac changes. Q J Med 1993; 86: 669- 675

17. Fine I, Brainerd H, Sokolow M. Myocarditis in Acute Infectious Disease. Circulation, Volume II, December 1950.

18. Kocak G, Tabel Y, Karakurt C, Demirdag Y Y. Electrocardiographic Abnormalities of Acute Pneumonia and Bronchiolitis in children. Inonu university Tip Fakultesi Dergisi 2009; 16(2) 6973

19. Bardi-Peti L, Ciotu E P. Pulmonary hypertension during acute respiratory disease in infants. Maedica 2010; 5(1): 13-19

20. Senocak F, Özkutlu S. Neonatal tricuspid insufficiency Doppler echocardiographic study of 49 cases. Cardiol Young 1995; 5: 172-175.

21. Neubauer $C$. Myocarditis in acute infectious disease. Arch Dis Child 1944; 19: 178.

22. Mahmoud M, El-Fouly M, Afifi M, Eraby A. Evaluation of cardiac changes in children with pneumonia. $5^{\text {th }}$ world Congress of Pediatric cardiology and cardiac surgery 26 June 2009.
23. Costard-Jackle A, Fowler M: Influence of preoperative pulmonary artery pressure on mortality after heart transplantation: testing of potential reversibility of pulmonary hypertension with nitroprusside is useful in defining a high risk group. J Am Coll Cardiol 1992; 19:48-54.

24. Douglas P, Bardy G, Worley S. Tachycardia induced cardiomyopathy; A reversible form of Left Ventricular Dysfunction. American Journal of Cardiology.1986; 57(8): 563-570

25. Nerheim P, Birger-Botkin S, Piracha L. Heart Failure and sudden death in patients with Tachycardia-Induced cardiomyopathy and Recurrent Tachycardia. Circulation 2004; 110:247-252

26. Shah R, Semigran M. Pulmonary Hypertension secondary to Left Ventricular Dysfunction: Contemporary Diagnosis and Management. Current Heart Failure Reports. 2008; 8(4): 226-232.

27. Butler J, Chomsky DB, Wilson JR: Pulmonary hypertension and exercise intolerance in patients with heart failure. J Am Coll Cardiol 1999; 34:1802-1806.

28. Costard-Jackle A, Fowler M: Influence of preoperative pulmonary artery pressure on mortality after heart transplantation: testing of potential reversibility of pulmonary hypertension with nitroprusside is useful in defining a high risk group. J Am Coll Cardiol 1992; 19:48-54.

29. Ghio S, Gavazzi A, Campana C, et al.: Independent and additive prognostic value of right ventricular systolic function and pulmonary artery pressure in patients with chronic heart failure. J Am Coll Cardiol 2001; 37:183-188.

30. Enriquez-Sarano M, Rossi A, Seward J. Determinants of Pulmonary Hypertension in Left Ventricular Dysfunction. J Am Coll Cardiol. 1997;29(1):153-159.

31. Pinamonti B, Di Lenarda A, Sinagra G. Restrictive left ventricular filling pattern in dilated cardiomyopathy assessed by Doppler echocardiography. clinical, echocardiographic and hemodynamic correlations and prognostic implications (Heart Muscle Disease Study Group). J Am Coll Cardiol. 1993; 22:808-815.

32. Guyton AC; Textbook of Medical Physiology. 7th ed. 1986 WB Saunders Philadelphia:287294. 
33. Porter TR, Taylor DO, Cycan A; Endotheliumdependent pulmonary artery responses in chronic heart failure. influence of pulmonary hypertension. J Am Coll Cardiol. 1993; 22: 1418-1424.

34. Burnett JC Jr, Kao PC, Hu DC; Atrial natriuretic peptide elevation in congestive heart failure in the human. Science. 1986; 231: $1145-1147$.

35. Stewart DJ; Endothelin in cardiopulmonary disease. Factor paracrine vs neurohumoral. Eur Heart J. 1993; 14 Suppl I: 48-54.
36. Cody RJ, Haas GJ, Binkley PF, Capers Q, Kelley R; Plasma endothelin correlates with the extent of pulmonary hypertension in patients with chronic congestive heart failure. Circulation. 85 1992:504-509.

37. Heikkila J, Karjaleinen J. Evaluation of mild acute infectious myocarditis. Br Heart J 1982; 47: 381-391.

38. N.Sreeram, J. G. Watson, S. Hunter. Cardiovascular effects of Acute Bronchiolitis. ACTA Paediatrica 1991; 80(1): 133-136. 